\title{
FORMACIÓN PROFESIONAL UNIVERSITARIA EN TURISMO EN LA REGIÓN LA LIBERTAD - PERÚ Y SU RELACIÓN CON LAS NECESIDADES FORMATIVAS DEL SECTOR TURISMO
}

\author{
UNIVERSITY PROFESSIONAL TRAINING IN TOURISM IN LA \\ LIBERTAD REGION - PERU AND ITS RELATIONSHIP TO THE \\ TRAINING NEEDS OF THE SECTOR TOURISM
}

\author{
Marcia Adriana Iberico Díaz \\ Universidad Nacional de Trujillo, Perú
}

\section{RESUMEN}

El conocimiento y la especialización contribuyen al desarrollo de la sociedad. El turismo aporta nuevos conocimientos para el avance del mundo, pero el adecuado desenvolvimiento y logro de esta actividad requieren una visión integral, holística y sistemática, para lo cual es necesario contar con capital humano calificado, especialmente en las labores de dirección. El objetivo de este artículo es determinar la relación entre la formación profesional en turismo que imparten las universidades de la Región La Libertad y las necesidades formativas del sector. Los métodos utilizados fueron el etnográfico y el analítico-sintético, aplicando entrevistas y encuestas a los responsables involucrados en el tema. Los resultados demuestran que las universidades de la región están enfocadas en una formación técnico-operativa y que el sector tiene una visión limitada de sus necesidades formativas reales respecto a las capacidades y competencias con las que se debe contar.

Palabras clave: Educación, formación universitaria, profesional en turismo, turismo.

\begin{abstract}
The knowledge and expertise contribute to the development of society. Tourism provides new insights for the advancement of the world, but for the adequate development and achievement of this activity it is necessary an integral, holistic and systematic view. Therefore, it is necessary to have a qualified human capital especially in management. The aim of this paper is to determine the relationship between vocational training provided by universities of tourism in La Libertad Region and the training needs of the sector. The methods used are ethnographic and analytic-synthetic, using interviews and surveys at the responsible sector. The results demonstrate that the universities in the region are focused on technical training-operational and that the sector has a limited view of their real needs regarding the capabilities and skills with which workers must have.
\end{abstract}

Key words: Education, university training, tourism professional, tourism 


\section{Introducción}

El desarrollo alcanzado por la sociedad se debe, en gran medida, al cúmulo de conocimientos y especializaciones existentes. El turismo es uno de los acontecimientos que viene contribuyendo al desarrollo del mundo actual, pero debe ser profesional, sistemático y adecuado a las expectativas; lo que no solo involucra a las empresas con miras a su desarrollo individual o a los estados como un colectivo, sino que termina siendo todo un sistema que implica a la comunidad anfitriona en la evaluación y aprovechamiento de sus recursos y atractivos turísticos, en la promoción de la actividad tanto por parte del sector público como del privado, y en la atención al tema de la superestructura e inversión en turismo, entre otros aspectos sustanciales.

Hoy en día el turismo reclama más atención, mayor especialización, un compromiso acorde a los conceptos y tendencias del desarrollo moderno, así como visión y comprensión amplias de la sociedad, de la cultura y del ambiente que lo rodea. Entonces ya no se trata solo de formar técnicos que maximicen la eficiencia y calidad de los servicios, sino profesionales que sean capaces de innovar, de modelar la captación de técnicas y conocimientos propios, que aporten al sistema y al entorno turístico alternativas viables de desarrollo, con formación científica y técnica que les permita idoneidad en el ejercicio profesional.

La formación que actualmente se imparte en las universidades, tanto en el Perú como en el mundo, es esencialmente práctica. Muchos de los responsables confunden el rol del turismo al plantear los planes curriculares, se centran en los modelos tradicionales de atención desatendiendo la integralidad de su gestión. La observación de la realidad que enfrenta el país -sobretodo en el norte-, la escasez de una competitiva planta de servicios turísticos, recursos en estado de desarrollo incipiente, un sector prometedor, una comunidad expectante por participar frente a una demanda ávida de nuevos y mejores destinos turísticos fueron las razones que llevaron a plantear esta investigación con el objetivo de determinar la relación existente entre la formación profesional en turismo que vienen impartiendo las universidades de la Región La Libertad con las necesidades del sector.

Para ello se analizaron los planes curriculares de las universidades de la región que forman profesionales en la especialidad, se identificó la orientación de esta formación y se sistematizó la relación entre las necesidades y exigencias que presenta el sector y la formación impartida. La hipótesis planteada fue que "la formación del profesional en turismo por las universidades de la Región La Libertad se relaciona limitadamente con las necesidades formativas del sector turismo".

La investigación se desarrolló en tres etapas: en la primera, de gabinete, se consultaron documentos y bibliografía que ayudaron a enfocar la problemática y a desarrollar la teoría pertinente; en la segunda se realizó trabajo de campo, con visitas a las universidades y a los responsables en turismo del sector público y privado para entrevistarlos y/o encuestarlos; finalmente, los datos y documentación resultantes se sistematizaron en relación con la hipótesis y los objetivos, para reflejar todo el proceso investigativo.

La escasez de antecedentes y de bibliografía no fue limitante para la investigación, ya que el acceso a fuentes primarias y la colaboración de los actores directos ayudaron al logro de las metas fijadas. En el proceso 
fue necesario analizar los planes curriculares de cada universidad y comparar los perfiles profesionales, se entrevistó a los responsables educativos y se consultó las impresiones de empresas e instituciones acerca de la formación de estos profesionales y de las necesidades formativas que tienen, con el fin de comparar y saber si responden a ellas en cuanto a las competencias y habilidades requeridas por el mercado laboral de esta zona.

Fueron analizadas tres escuelas universitarias, elegidas por su antigüedad, considerándose indispensable haber tenido por lo menos una promoción egresada, lo que permitió evaluar el plan curricular terminado. En esta evaluación participaron miembros de los organismos públicos y privados, aplicándose métodos como el etnográfico y el analítico-sintético para recoger y sistematizar la información; técnicas como la observación directa, la entrevista y la encuesta, e instrumentos como el cuestionario y la guía de entrevista.

La presente es una investigación aplicada, no experimental, de estudios de correlación entre las necesidades formativas del sector turismo y la formación profesional proporcionada por las universidades de la Región La Libertad. Las unidades de análisis no han sido alteradas (ex-post facto) y contrastadas en la realidad para identificar las necesidades profesionales del sector turismo y cómo las universidades las atienden con sus planes de estudio; el desarrollo fue a un nivel exploratorio-correlacional pues se examina a profundidad la formación profesional en turismo en la región. Mediante una muestra aleatoria y de elección intencional se logró establecer la relación entre la formación profesional y las necesidades formativas del sector.

Se trabajó con tres de las universidades de la región que cuentan con carreras de turismo: la Universidad Nacional de Trujillo, la Universidad Católica de Trujillo y la Universidad César Vallejo, fundamentalmente porque imparten diversos modelos de formación profesional y tienen más de cinco años de funcionamiento, la población muestra entrevistada fueron tres directores de las carreras de Turismo de estas universidades y 52 autoridades de instituciones públicas y privadas. El resto de universidades es de creación reciente y su influencia en la gestión del turismo no se puede determinar con certeza. Uno de los instrumentos claves fue la encuesta que se aplicó a directivos de instituciones y empresas demandantes de profesionales, conformada por doce preguntas: tres de carácter valorativo, tres para establecer prioridades, dos sobre las habilidades y competencias profesionales, tres sobre el sector formativo y una sobre el potencial que tienen para ocupar puestos laborales vacantes.

\section{Resultados}

El progreso de un individuo está vinculado al conocimiento, para lo cual es fundamental la educación que lo prepara desarrollando sus potencialidades hasta alcanzar el mejor desempeño posible, permitiéndole dominar la naturaleza. Uno de los niveles educativos es el universitario que lleva a la persona a su reconocimiento como profesional. El ser de la universidad se encuentra estrechamente vinculado con el ser del hombre, con su formación integral y su misión ante la sociedad. No se puede confundir a la universidad con un lugar donde el estudiante se convierta en depósito de conocimiento. La universidad ha de ser la custodia de la verdad y de la ciencia, al margen de posturas ideológicas. Es la institución generadora de pensamiento propio, transformador 
y con proyección en orden de la vida social... espejo del pensamiento libre... debe trascender el campo de lo académico, pedagógico y científico, promoviendo el despertar de la imaginación creadora, como parte integral de la formación activa (Parent, Farrand, Esquivel, Soriano \& Pérez, 1994).

Las universidades, para lograr su misión, hacen del currículo la herramienta básica del proceso educativo. Según Campos (2008: 136), citando a Arnaz, el currículo es "el plan que norma y conduce explícitamente un proceso concreto y determinante de enseñanza-aprendizaje que se desarrolla en una institución educativa". Para Iafrancesco(2004: 24) es el conjunto de criterios, planes de estudio, programas, metodologías y procesos que contribuyen a la formación integral y a la construcción de la identidad cultural nacional, regional y local, incluyendo los recursos humanos, académicos y físicos para poner en práctica las políticas y llevar a cabo el proyecto educativo institucional. Dentro del currículo, el perfil profesional es el vértice que orienta y sistematiza las funciones y tareas del desempeño profesional de una persona. En opinión de Campos (Ibíd.: 152) se trata de "una descripción de las características que se requieren del profesional para abarcar y solucionar las necesidades sociales... el perfil profesional lo componen tanto conocimientos y habilidades como actitudes".

El turismo es una actividad reciente en la vida de los hombres, producto de todos los cambios que se han suscitado en la sociedad contemporánea. La formación profesional en esta materia ha tenido que adaptarse a las concepciones de las diferentes épocas por las que ha atravesado el hombre desde que se concibiera al turismo como tal. Es más que el simple desplazamiento de personas, lleva consigo la idea de la regeneración, de la integralidad del ser humano y del respeto hacia todo lo que le acoge y le rodea. La Agencia Nacional de Evaluación de la Calidad y Acreditación (ANECA, 2004: 29) diferencia tres períodos en la evolución del turismo:

- En un primer período se le concibe como una actividad productiva menor, un tiempo inútil opuesto al trabajo y por lo tanto con escaso eco en la comunidad científica. Se trataba solo de cuestiones operativas y el aprendizaje era eminentemente práctico.

- En un segundo período se le considera como el punto de encuentro entre distintas disciplinas (Ibíd.: 30); es decir, traspasa el ámbito de la moda, de las masas y se convierte en un punto de preocupación que merece un tratamiento interdisciplinario. Es una actividad con implicaciones económicas, geográficas y sociales.

- En un tercer período es visto como un objeto de análisis especializado (Ibíd.: 32), como una unidad estructural que forma parte indiscutible del modo de vida de la sociedad y de la economía de los países del sistema capitalista. La complejidad del tratamiento que requiere el turismo obliga, por lo tanto, a que aumente el número de disciplinas que lo incorporan como objeto de estudio, hecho que lleva a tener una visión más profesional de estudio. En ese marco, la universidad tiene la responsabilidad de asumir el reto de formar profesionales con capacidades y competencias necesarias para desarrollarlo.

En las investigaciones sobre este tema no se ha considerado la necesidad urgente de enfatizar que la formación profesional debe tener un enfoque y una perspectiva integrales y, sobre todo, humanos. Los criterios con que se forman los profesionales para afrontar con éxito las necesidades que la realidad exige a esta 
profesión, muchas veces se idealizan, se teorizan e incluso se ideologizan desde el punto de vista académico, pero estas percepciones no siempre son las adecuadas y conllevan errores y defectos que deforman la realidad y el normal desenvolvimiento de las actividades humanas.

El avance de la ciencia, la tecnología, la informática y la globalización; los cambios en las formas de viajar; la especialización de la hospitalidad y la presencia de atractivos turísticos en lugares con ecosistemas frágiles; el impacto de los visitantes sobre el medioambiente y la sociedad; y la competitividad que requiere cada destino turístico exigen una formación altamente especializada en los profesionales de turismo. Se requiere además una visión integral y sistémica, de modo que el manejo de un destino sea competitivo y sostenible. El sistema turístico debe ser tratado como un todo ya que implica un trabajo integrado y coordinado -horizontal y verticalmente-, entendiéndose a dicho sistema como el "conjunto de elementos (superestructura, demanda, comunidad local, atractivos, equipamiento e infraestructura) interrelacionados que proporcionan satisfacción a las necesidades de uso del tiempo libre" (Boullón, Molina \& Rodríguez, 2006: 17).

El sistema turístico, por lo tanto, se convierte en el soporte de la gestión para el desarrollo de un producto competitivo, y la mejor garantía de este desarrollo es contar con profesionales probos. Juan Lucero (2006) afirma que se requiere actores que comprendan sus implicancias no solo económicas, sino también culturales, políticas y geopolíticas, sociales, ambientales y espirituales; y, en concordancia con la visión de la Organización Mundial de Turismo (OMT), afirma que el gran reto de la formación es superar el tratamiento metodológico parcelado y atomizado, proponiendo una educación integral para lograr un cuerpo de conocimientos interdisciplinario y plural, en una perspectiva conjunta (Ibíd.).

Durante las dos últimas décadas del siglo XX el turismo entra con mayor fuerza al mundo académico. En el ámbito nacional, a partir de los años noventa, se multiplicaron las universidades involucradas en la formación en turismo, asumiendo el reto de crear planes de estudio con miras a formar profesionales que sean capaces de llevar adelante esta actividad, dejando atrás la formación técnica que solo requería tres años de preparación; sin embargo, el enfoque aún mantiene un gran peso técnico-operativo, preparando profesionales básicamente para atención en hoteles, restaurantes y agencias de viajes.

\section{Las universidades y la formación en turismo en la Región La Libertad}

Las carreras de turismo en las universidades estudiadas han venido desarrollándose en esta zona del país desde 1993 (Tabla 1), mostrando una gestión limitada ya que los recursos humanos, materiales y económicos dependen de la administración central de cada universidad, lo que les resta autonomía en la toma de decisiones respecto a la infraestructura y al equipamiento que deben presentar. Por ende, disminuyen los niveles de eficacia y eficiencia debido a una formación con escasa intervención en el desarrollo de este sector.

De la Universidad Nacional de Trujillo (UNT) han egresado 560 profesionales, 20 de la Universidad Católica de Trujillo Benedicto XVI (UCT) y 25 de la Universidad César Vallejo (UVC). La mayoría de los egresados de estas dos últimas universidades se desempeñan en cargos operativos en empresas turísticas, a diferencia 
de los egresados de la UNT, muchos de los cuales han asumido cargos gerenciales en diferentes instituciones públicas y privadas, o se han involucrado en consultorías, en investigación y en el ámbito académico.

Tabla 1

Carrera profesional según universidad, facultad y año de creación

\begin{tabular}{lllc}
\hline Universidad & \multicolumn{1}{c}{ Facultad } & \multicolumn{1}{c}{ Escuela Profesional } & Año de creación \\
\hline Universidad Nacional de Trujillo & Ciencias Sociales & Escuela Académico Profesional de Turismo & 1993 \\
Universidad Católica de Trujillo & Administración y Finanzas & Carrera Profesional de Administración Turística y Hotelera & 2006 \\
Universidad César Vallejo & Ciencias Empresariales & Escuela de Administración en Turismo y Hotelería & 2007 \\
\hline
\end{tabular}

Fuente: Registro de entrevista, mayo de 2012.

Los currículos que vienen desarrollando las carreras de turismo en estas universidades se diferencian en el enfoque formativo: en la UCT y en la UCV se orientan al ámbito técnico-operativo para empresas, mientras que la UNT presenta una visión de gestión-técnica-operativa que vincula el aspecto social (Tabla 1), las empresas y las instituciones reguladoras del sector. Esta condición se nota en las titulaciones que ofrecen: la UNT otorga el título de licenciado en turismo, la UCT titula en gerencia de servicios turísticos y hoteleros, y la UCV otorga licenciaturas en administración en turismo y hotelería. Todas estas carreras tienen 58 cursos en sus planes de estudio, la UNT y la UCT con 220 créditos y la UCV con 210. De estas titulaciones anotamos el peso académico que se asigna a los cursos: la UNT visualiza el turismo completo, considerando cursos de planificación, gestión y operación, añadiendo a ello una notable dosis de investigación que permite a los graduandos familiarizarse con la realidad y proponer alternativas de desarrollo ante la problemática que afrontan; la UCT y la UCV entrenan a los graduandos en el campo empresarial con miras a un crecimiento de la planta turística. Sin embargo, las tres universidades presentan deficiencias en infraestructura y equipamiento, por lo que su entrenamiento en el campo operativo se ve afectado.

Al ser consultados los integrantes del sector público y privado vinculados al turismo acerca de su visión de la formación universitaria en relación a los cargos que los egresados deben ocupar, el 52\% opina que los licenciados o profesionales en turismo debe tener la prioridad para manejar el sector (Figura 1), seguidos con relativa cercanía por la "gente con experiencia en el sector", lo que implica que se prioriza el conocimiento y la formación profesional. En cuanto a los ámbitos de gestión, el 40\% considera que los profesionales en turismo deben ocuparse de la gestión de instituciones del sector, reforzando la necesidad de cuadros profesionales (Figura 2). Sin embargo, es preciso resaltar que el 50\% prioriza la gestión empresarial como campo de acción de los profesionales, mientras que el 10\% considera la necesidad de desarrollar el campo académico y de investigación o de desarrollo del conocimiento. 
Figura 1. Orden de prioridad del tipo de profesional que debe manejar el turismo según miembros del sector

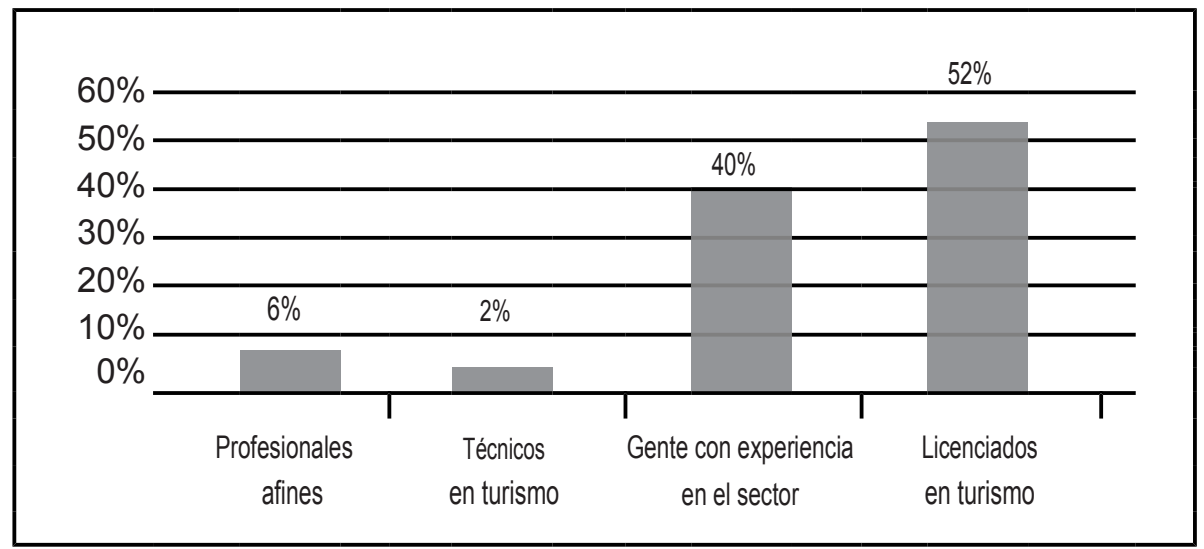

Fuente: Registro de encuesta, mayo y junio de 2012.

Figura 2. Orden de prioridad de los ámbitos laborales en los que se debe desempeñar el profesional en turismo en la Región La Libertad según miembros del sector

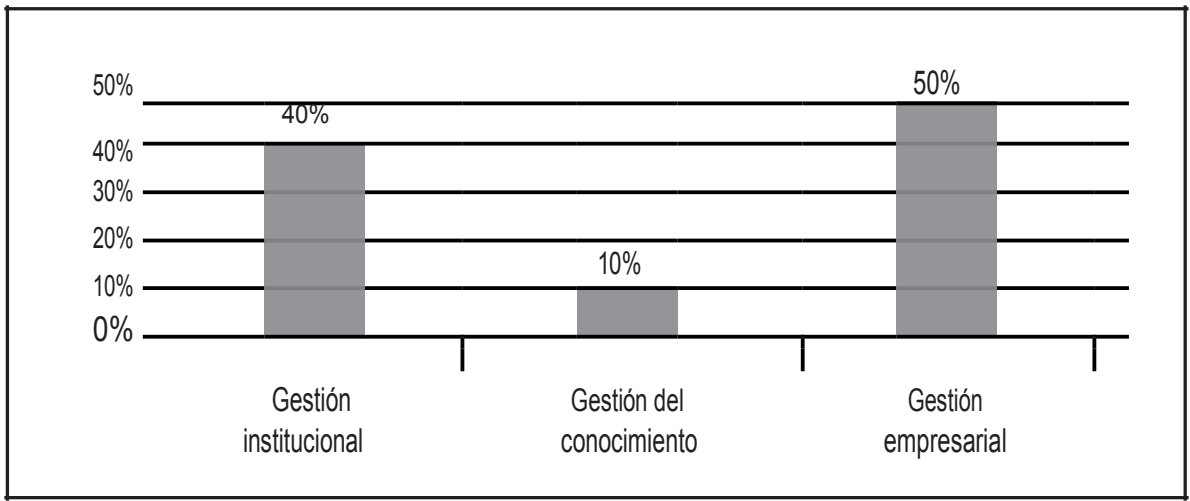

Fuente: Registro de encuesta, mayo y junio de 2012.

Las empresas son el campo más amplio en el que se desarrolla la actividad turística; sin embargo la Región La Libertad, aun cuando constituye uno de los polos empresariales más consolidados del norte peruano, no cuenta con una planta turística amplia y significativa, por lo que la gestión de empresas se convierte en una prioridad, no solo desde el punto de vista operativo, sino también desde el punto de vista de la proyección y de la dirección y canalización de inversiones que lleven a crear empresas competitivas; por ello, el 50\% de los encuestados indica esa necesidad. La gestión institucional implica también la gestión de los atractivos turísticos, de las organizaciones de base y de la superestructura turística; en suma,de todo aquello que priorice al turismo como motor del desarrollo local y regional. Así, los egresados están llamados a gestionar desde un recurso turístico hasta la complejidad que implica el manejo de un destino. 
En cuanto al orden de prioridad en los niveles jerárquicos, el profesional en turismo debe estar capacitado para los niveles de gestión por sobre los de administración, entendiendo la gestión como la apuesta, iniciativa y emprendimiento para llevar adelante una idea o proyecto. En ese sentido, el 58\% de los encuestados considera que los niveles de gestión deben ser ocupados por estos profesionales, el 27\% opina que los puestos operativos corresponden a los profesionales en turismo; mientras que el $15 \%$ plantea como segunda opción los puestos de administración, entendida esta como una profesión específica. Por lo tanto un administrador o una jefatura no son la prioridad en el sector, lo que se necesita es recurso humano que desempeñe las funciones para las que ha sido preparado (Figura 3).

Figura 3. Orden de prioridad de los niveles jerárquicos para los que deben estar capacitados los profesionales en turismo según miembros del sector

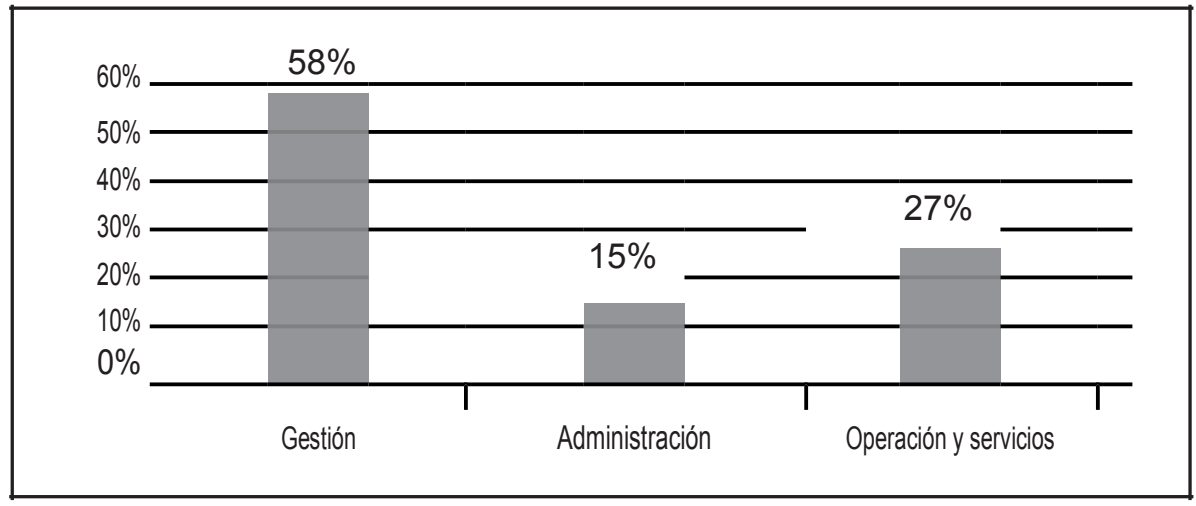

Fuente: Registro de encuesta, mayo y junio de 2012.

Figura 4. Percepción del cumplimiento del desempeño profesional de los egresados universitarios en relación a las necesidades del turismo según miembros del sector

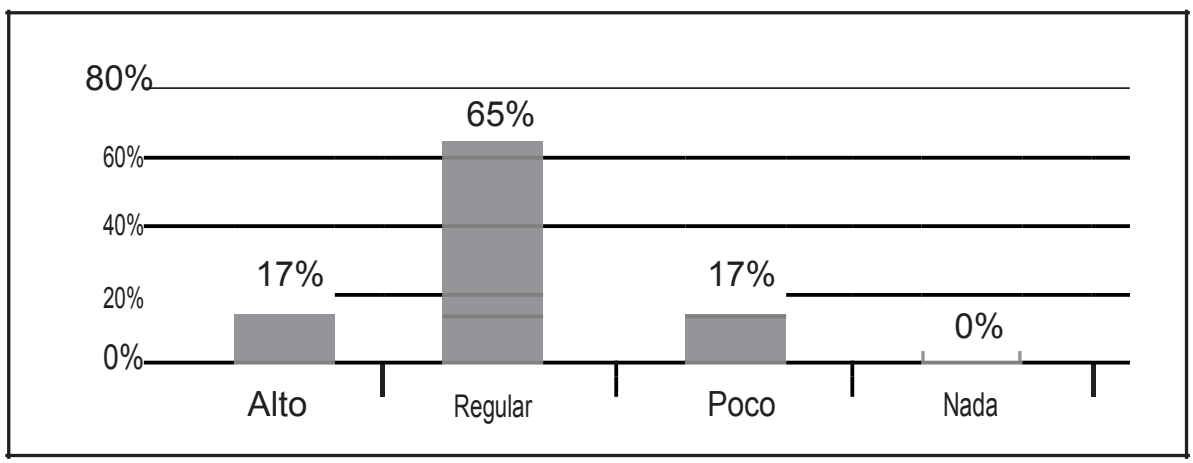

Fuente: Registro de encuesta, mayo y junio de 2012.

Al ser consultados los miembros del sector sobre su percepción del desempeño profesional en los puestos asignados a los egresados universitarios, el 17\% considera que el rendimiento es alto u óptimo; sin embargo, hay un $65 \%$ que califica este rendimiento como regular, lo que está en relación con los diferentes puestos 
asignados tanto en las instituciones públicas como en las privadas. Al desagregar los resultados por universidades, la UNT, con mayor presencia en el sector, resulta ser la mejor calificada, en tanto que las otras dos muestran un desempeño con menores calificaciones.

Además se hizo la consulta sobre los puestos asignados, a lo que respondieron que vienen ejerciéndose básicamente a nivel operativo, seguidos por mandos medios y por último en puestos de toma de decisiones; debido esencialmente a la formación que vienen recibiendo en las instituciones universitarias. Sin embargo, cuando se refieren a los puestos que esperan asignarles, estos se orientan a niveles de mandos gerenciales y toma de decisiones en las diferentes instituciones. También se les solicitó que opinaran sobre las competencias tanto generales como específicas que deben presentar estos profesionales. Entre las competencias generales se mencionó que deben tener "preocupación por la calidad" (77\%), "iniciativa y espíritu emprendedor" (77\%), "capacidad para aplicar los conocimientos en la práctica" (65\%) y "motivación por el logro" (67\%), como se observa en el Figura 5. Entre las competencias específicas exigidas encontramos: "desarrolla las actividades profesionales con ética, honestidad y responsabilidad" (77\%), "respeta las costumbres, culturas, valores y

Figura 5. Nivel de importancia de las competencias generales que deben presentar los

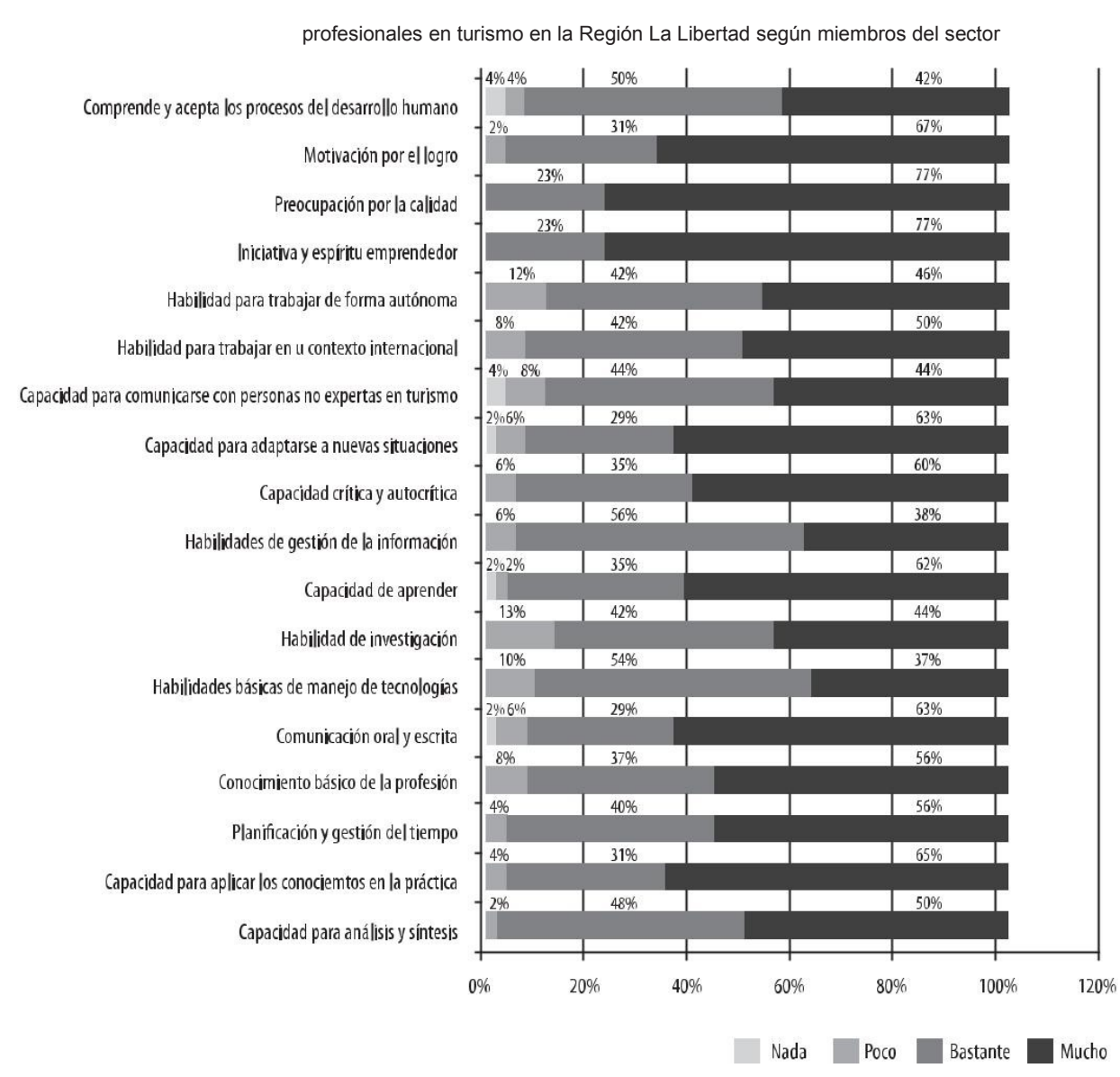

Fuente: Registro de encuesta, mayo y junio de 2012. 
tradiciones de la comunidad receptora y del turista" (75\%), "demuestra liderazgo y busca la mejora continua en el servicio, es agente de cambio en la actividad turística" (69\%) y "crea, planifica, dirige y evalúa el funcionamiento de empresas turísticas" (62\%); todo lo cual se ve reflejado en el Figura 6.

Figura 6. Nivel de importancia de las competencias específicas que deben presentar los profesionales en turismo en la Región La Libertad según miembros del sector

Respeta las costumbres, culturas, valores y tradiciones de la comunidad receptora y del turista Desarrolla las actividades profesionales con ética, honestidad y responsabilidad

Promueve la conciencia turística con capacidad pedagógica

Es líder, capaz de generar actitudes válidas de identificación organizacionaly de participación Valora y fomenta la identidad, el patrimonio cultural y natural, y lidera eldesarrollo y bienestar colectivo Demuestra liderazgo y busca la mejora continua en el servicio, es agente de cambio en la actividad turística Es dinámico y proactivo desarrollando una atención y servicio de excelencia Posee habilidades de intervención y empatía en la comunidad y ambientes donde trabaja Conoce y aplica procedimientos operativos en las empresas turísticas según su rubro Capaz de gestionar y administrar los recursos financieros en la actividad turistica Capaz de dirigir y gestionar el recurso humano Conoce y utiliza materiales y herramientas tecnológicas en su desempeño laboral Dominio de idiomas

Es creativo e innovador para desarrollar ideas encaminadas a su desempeño laboral Desarrolla planes y politicas en materia turística Identifica problemas, propone alternativas y toma decisiones en la actividad turistica Maneja las relaciones interpersonales y públicas en la actividad turistica Organiza, dirige e implementa atractivos turísticos Participa y lidera grupos interdisciplinarios para formular, ejecutar y evaluar proyectos turisticos Crea, planifica, dirige y evalúa el funcionamiento de empresas turísticas Está preparado para ocupar los diversos niveles jerárquicos de una organización pública o privada Comprende el funcionamiento de los destinos, estructuras turisticas y sus sectores empresariales Conoce e identifica las características, procesos históricos y de desarrollo de los espacios turísticos Conoce, interpreta y utiliza las diferentes políticas y marco legal de la actividad turística Identifica la problemática del sector y aplica los conocimientos conducentes a resolverlos Diseña e implementa actividades de marketing para la gestión y comercializacion de productos y servicios Posee capacidades para poner en valor y comercializar destinos y productos turísticos Conoce y determina el potencial de los recursos turísticos naturales y culturales del país Analiza y evalúa los impactos económicos, sociales, culturales y ambientales generados por el turismo Conoce métodos y técnicas para planear, implementar y dirigir propuestas de investigación y desarrollo Identifica, reconoce y utiliza conceptos, categorías, teorías y terminologías de turismo Desarrolla una gestión de calidad en organizaciones públicas y privadas Analiza y evalúa el sistema turístico en el marco global

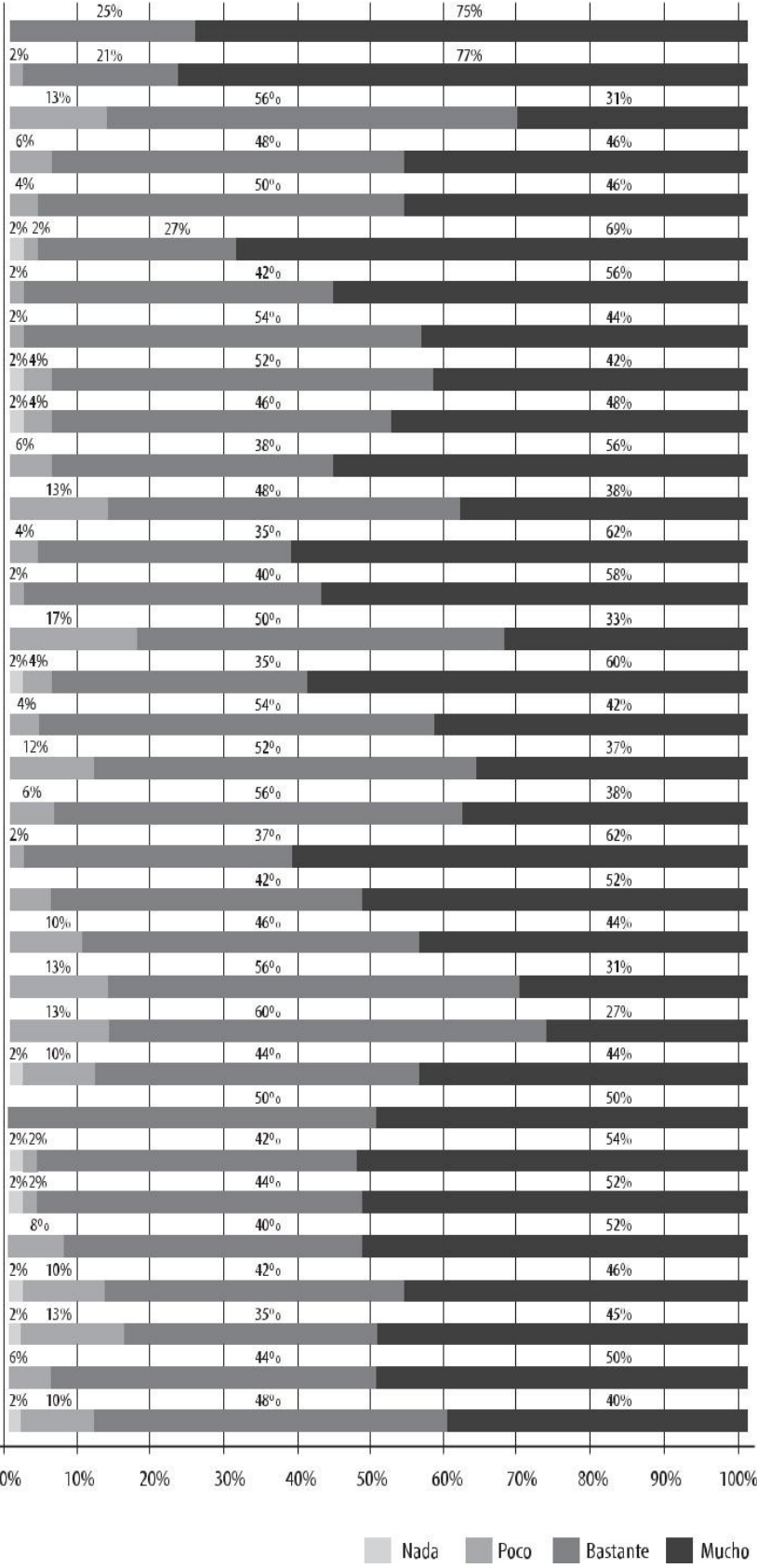

Fuente: Registro de encuesta, mayo y junio de 2012. 
La universidad peruana aún no ha salido del todo de la primera fase respecto a la formación y tímidamente se introduce en la segunda, las carreras turísticas están íntimamente ligadas a la administración y a la economía (Tabla 1), en sus planes de estudio se puede apreciar la tendencia a los servicios de hotelería y focalizan la formación en la parte operativa de las empresas turísticas; exceptuando a la UNT que sale de este esquema y lo enfoca como un proceso con perspectiva integral.

La formación profesional universitaria está llamada a articular de manera dialéctica el conocimiento teórico con la práctica, base para el logro de una cultura académica comprometida con la investigación, la transformación y el desarrollo de una realidad, donde el profesional aprende a pensar cooperativamente o por sí mismo a través del diálogo constructivo sustentado en el compromiso ético. La universidad es la responsable de cristalizar este compromiso respondiendo al desarrollo con perfiles profesionales acordes a la realidad. En el caso del turismo regional se necesita preparar un producto competitivo y posicionarlo, para lo cual el sector requiere que las escuelas profesionales formen ciertas competencias generales en cuanto al manejo de esta actividad.

De acuerdo a los miembros del sector, las competencias generales que priorizan son la "preocupación por la calidad"; es decir, el sector es consciente de que la prioridad en turismo es la "calidad" como el concepto más constante. Del mismo modo son prioritarios la "iniciativa y espíritu emprendedor", seguidos de la "motivación por el logro". Cabe enfatizar que estas competencias se requieren tanto para la "gestión empresarial" como para la "gestión institucional"; sin embargo, las universidades privadas priorizan al sector empresarial, como se puede apreciar en sus planes curriculares, buscando la competitividad de la empresa de manera particular y dejando muchas veces de lado la competitividad como destino turístico. Como se puede apreciar, las competencias generales más requeridas por el sector tienen más relación con la actitud del profesional y con el criterio para resolver situaciones muy sensibles al desarrollo turístico, para lo cual no solo es necesaria una actitud mecánica, sino una actitud crítica y de convicción de lo que se está realizando.

En cuanto a las competencias específicas mencionadas la más importante fue: "crea, planifica, dirige y evalúa el funcionamiento de empresas turísticas" (62\%), lo que se explica porque la región en general busca elevar su competitividad mediante el desarrollo de inversiones con criterios de calidad, para lo cual resulta clave el recurso humano profesional.

El mismo sector resalta la importancia de aspectos que tienen que ver con la visión de desarrollo del destino turístico, del cual las empresas son componentes que contribuyen de manera significativa a la valoración y la calidad del producto turístico. Asimismo, es necesario resaltar que el tipo de profesional de turismo hoy en día debe ser proactivo, emprendedor, humano con capacidad de liderazgo y, sobre todo, que sepa trabajar en equipo, que tenga vocación de servicio y promueva el valor del patrimonio cultural y natural. De esa manera la formación profesional universitaria tendrá nuevos giros para construir un modelo científico del turismo y garantizar el desarrollo turístico de esta zona.

Es preciso entender que el sector turismo no está conformado únicamente por las empresas turísticas y menos por solo la hotelería u otro enfoque sesgado: engloba todo el aparato que dinamiza, desde sus bases 
sociales correspondientes a la comunidad, su parte operativa o de servicios y economía, y la parte organizativa y formativa. No podemos negar que las comunidades miran actualmente al turismo como una alternativa de desarrollo económico, mientras que los estados lo visualizan como una alternativa de desarrollo social. En tanto la academia especializada apuesta por un desarrollo integral, cultural y ambientalmente sensato; sin embargo, al momento de planificar los estudios y formación no se realiza un análisis consciente de la realidad turística.

\section{Conclusiones}

1. La formación profesional en turismo que vienen impartiendo las universidades de la Región La Libertad se relacionan limitadamente con las necesidades formativas del sector, como consecuencia del poco conocimiento real de estas necesidades.

2. La formación profesional en turismo que imparten las universidades en la Región La Libertad tiene un enfoque técnico-operativo, poco de gestión empresarial, casi nula de gestión de destinos y escaso manejo del desarrollo del sector productivo con una visión sistémica y holística.

3. El planteamiento de las carreras de turismo respecto a los centros laborales o de prácticas a los cuales pueden dirigirse los alumnos y egresados no responde a los perfiles profesionales que tienen; por lo tanto, los planes de estudios universitarios no reflejan lo que realmente aspiran los estudiantes.

4. Las demandas productivas del sector turístico en cuanto a la formación del recurso humano exigen que los profesionales demuestren condiciones para gestionar, gerenciar y operativizar los destinos y empresas turísticas, según se puede apreciar en las competencias generales y específicas determinadas por sus miembros (Figuras 5 y 6 ).

\section{Referencias}

ANECA (2004). Libro Blanco del Título de Grado en Turismo. Madrid: Agencia Nacional de Evaluación de la Calidad y Acreditación.

Boullón, R., Molina, S. \& Rodríguez, M. (2006). Un Nuevo Tiempo Libre. México: Trillas

Campos, S. (2008). Currículo universitario por competencia. Arequipa: Lemoine.

Iafrancesco, G. (2004). La evaluación integral y de los aprendizajes desde la perspectiva de una escuela transformadora: Contexto, concepto, enfoque, principios y herramientas. Recuperado de http://www.colombiaaprende.edu.co/html/mediateca/1607/articles174388 archivo.pdf

Lucero, J. (2006). Seminario Internacional Formación de Profesionales para el Desarrollo del Turismo y la Hotelería. Cómo preparar líderes para el cambio en el sector. Formación y competencias del profesional en turismo y hotelería. La experiencia argentina. Lima, Universidad de San Martín de Porres.

Parent, J. M., Farrand, J., Esquivel, N., Soriano, R. \& Pérez, E. (1994). ¿Qué es la Universidad? Recuperado de http://abulico.zzl.org/ v/uni01.htm 